\title{
Empfehlungen zur ethischen Unterstützung in der Medizin
}

Schweizerische Akademie der Medizinischen Wissenschaften (SAMW)
Korrespondenz:

lic. iur. Michelle Salathé Schweizerische Akademie der Medizinischen Wissenschaften (SAMW)

Petersplatz 13

CH-4051 Basel

Tel. 0612699030

m.salathé[at]samw.ch
An seiner Sitzung vom 29. Mai 2012 hat der Senat der Schweizerischen Akademie der Medizinischen Wissenschaften (SAMW) die definitive Fassung der Empfehlungen «ethische Unterstützung in der Medizin» verabschiedet. Die Subkommission unter dem Vorsitz von Prof. Samia Hurst aus Genf hat rund drei Jahre an diesen Empfehlungen gearbeitet, deren Ziel es ist, zur Qualität und Nachhaltigkeit der klinisch-ethischen Entscheidungsfindung beizutragen. Die Empfehlungen enthalten praktische Hinweise für bereits bestehende Angebote, sie richten sich aber auch an Institutionen, die ein solches Angebot aufbauen möchten. Die Empfehlungen umfassen alle Formen der ethischen Unterstützung: klinische Ethikkommission, Ethikkonsil, Ethikforum usw. len Stellungnahmen wurden die Ausarbeitung von Empfehlungen zur ethischen Unterstützung ausdrücklich begrüsst und die Empfehlungen als hilfreich beurteilt. Über den Empfehlungscharakter waren die Meinungen allerdings geteilt; teilweise wurde eine stärkere Verbindlichkeit gefordert. Am häufigsten wurde der ursprüngliche Titel «Strukturen zur ethischen Unterstützung in der Medizin» kritisiert. Diese Kritik ist nun in der definitiven Fassung berücksichtigt worden. Da es in der deutschen (und französischen) Literatur keinen etablierten Begriff gibt, der die explizite Entwicklung und Förderung ethischer Kenntnisse, Fähigkeiten und Haltungen in der Praxis von Institutionen des Gesundheitswesens umfassend beschreibt, wird in Anlehnung an das im

\section{Die Empfehlungen enthalten praktische Hinweise für bereits bestehende Angebote, sie richten sich aber auch an Institutionen, die ein Angebot aufbauen möchten.}

Die SAMW hat sich bewusst für «Empfehlungen» und nicht für «Richtlinien» entschieden, obwohl diese eine geringere Verbindlichkeit haben. Die Empfehlungen wollen Grundvoraussetzungen definieren, die zu erfüllen sind, damit eine hilfreiche ethische Unterstützung möglich ist. Sie betonen, dass der Einbezug einer ethischen Unterstützung kein Ersatz für die im Alltag notwendigen ethischen Diskussionen innerhalb eines Teams ist, aber in ethisch schwierigen Situationen zusätzliche Hilfe bieten kann. Bei der Ausarbeitung dieser Empfehlungen hat sich die Subkommission sowohl auf bereits bestehende internationale Empfehlungen zur Arbeit von Ethikstrukturen [1] gestützt als auch die aktuelle Literatur berücksichtigt. Mit einem Expertenworkshop am 29. Juni 2011, zu dem Mitglieder von bestehenden klinischen Ethikkommissionen eingeladen wurden, hat sie zudem auch spezifisch schweizerische Erfahrungen einbezogen.

In der Vernehmlassung von Anfang Dezember 2011 bis Ende Februar 2012 sind 58 Stellungnahmen zum Empfehlungsentwurf eingetroffen. Die SAMW hatte den angefragten Institutionen (Spitäler, Gesundheitsdirektionen, Fachgesellschaften, Ethikstrukturen) einen Fragebogen zur Verfügung gestellt. Dieser wurde von 46 Institutionen ausgefüllt und teilweise ausführlich kommentiert. Praktisch in al-
Englischen verwendete «ethics support» von ethischer Unterstützung gesprochen.

Die Subkommission hat die im Rahmen der Vernehmlassung eingebrachten weiteren Überarbeitungsvorschläge in einer ganztägigen Sitzung diskutiert und die Empfehlungen, gestützt auf diese Diskussion, überarbeitet. Das Kapitel «Anforderungen an Ethikstrukturen für spezifische Bereiche» wurde aus dem Anhang (der nur auf der Website veröffentlicht wird) in die Empfehlungen übernommen und durch einen Abschnitt zur Gefängnismedizin ergänzt. Schliesslich wurde das Kapitel Qualität umfassend revidiert. Im Anhang bleibt die Beschreibung der zurzeit verwendeten Methoden der ethischen Unterstützung. Die Empfehlungen halten dazu fest, dass sich bis heute keine Methode als den anderen überlegen ausgezeichnet hat und in diesem Bereich Forschungsbedarf besteht.

\section{Literatur}

1 Vgl. z. B. American Society for Bioethics and Humanities (ASBH). Core Competencies for Health Care Ethics Consultation. Improving Competencies in Clinical Ethics Consultation: An Education Guide. 2009 (www.asbh.org/); Akademie für Ethik in der Medizin (AEM): Standards für Ethikberatung in Einrichtungen des Gesundheitswesens. Ethik Med. 22(2):149-53. 


\title{
Empfehlungen der Schweizerischen Akademie der Medizinischen Wissenschaften (SAMW) Ethische Unterstützung in der Medizin
}

\begin{abstract}
Zusammenfassung
Die SAMW empfiehlt in diversen Richtlinien eine ethische Unterstützung [1] in der Medizin. In der Schweiz wurden in den letzten Jahren zahlreiche entsprechende Angebote entwickelt. Diese reichen von der Einzelfallberatung bis zur Ausarbeitung von ethischen Leitlinien oder der Organisation von Weiter- und Fortbildungsveranstaltungen.

Strukturen zur ethischen Unterstützung (Ethikstrukturen) sind jedoch nur dann hilfreich, wenn sie ihre Aufgaben adäquat erfüllen können. Die Empfehlungen «Ethische Unterstützung in der Medizin» formulieren in der Einleitung zum einen Ziele und Aufgaben solcher Strukturen, sie machen zum anderen aber auch auf damit zusammenhängende Grenzen, Gefahren und Missverständnisse aufmerksam.

In den Empfehlungen wird einerseits auf die Vielfalt der Strukturen und Methoden hingewiesen und auf einige Grundfragen eingegangen, welche die Kernelemente der Arbeit betreffen: Wann ist eine Anfrage für eine ethische Unterstützung sinnvoll? Was ist bei Einzelfallentscheidungen, beim Erstellen von Ethik-Leitlinien oder in der Aus-, Weiter- und Fortbil-
\end{abstract}

dung zu beachten? Andererseits werden Hinweise zur Etablierung von Ethikstrukturen gegeben. Welche strukturellen Voraussetzungen sollten erfüllt sein? Wie kann die Unabhängigkeit der Ethikstruktur gewährleistet werden? Welche Grundeinstellungen, Kenntnisse und Kompetenzen sollten ihre Mitglieder mitbringen? Wie sollte sie zusammengesetzt sein, und was trägt zur Erhöhung ihrer Sichtbarkeit bei? Zudem werden praktische Hinweise in Form einer Checkliste gegeben, die eine Hilfestellung zur Auswahl der geeigneten Struktur für die ethische Einzelfallberatung und zur Organisation der Beratungsprozesse bieten sollen.

Ethikstrukturen wurden primär in den grösseren Akutspitälern etabliert, kommen aber zusehends auch in anderen Bereichen zur Anwendung, sowohl im stationären als auch im ambulanten Bereich. In den vorliegenden Empfehlungen wird daher auch beschrieben, welche Anforderungen die Ethikstrukturen in den einzelnen Bereichen erfüllen sollten. Im Anhang werden diverse Methoden der ethischen Unterstützung (inkl. weitergehende Literaturhinweise) kurz vorgestellt.

\section{Einleitung}

Der medizinische Alltag stellt alle Beteiligten vor vielfältige ethische Herausforderungen. Dazu gehören beispielsweise Entscheidungen über das Fortführen oder den $\mathrm{Ab}$ bruch von lebenserhaltenden Massnahmen bei schlechter Prognose, die Respektierung des Selbstbestimmungsrechts der Patienten [2] oder die gerechte Verteilung der Ressourcen unter dem Druck der steigenden Gesundheitskosten. In allen genannten Situationen können Wertekonflikte entstehen. Medizin-ethische Richtlinien und Empfehlungen, wie diejenigen der Schweizerischen Akademie der Medizinischen Wissenschaften, formulieren Leitlinien und verstehen sich als eine Unterstützung für die Mitglieder von Behandlungsteams. Richtlinien und Empfehlungen können jedoch lediglich generelle Überlegungen beinhalten. In konkreten Fällen müssen die allgemeinen Standards der individuellen Situation angepasst werden. Die ethische Unterstützung in verschiedenen Formen kann in diesen Entscheidungsprozessen hilfreich sein.

Wie in vielen anderen Ländern etablieren sich auch in der Schweiz zunehmend Strukturen zur ethischen Unterstützung in der Medizin (Ethikstrukturen). Auch die SAMW empfiehlt den Beizug von ethischer Unterstützung in diversen Richtlinien.
Aufgrund dieser Entwicklung hat die SAMW eine Bestandesaufnahme der Angebote zur ethischen Unterstützung in der Medizin vorgenommen [3]. In den vorliegenden Empfehlungen [4] definiert sie nun, welche Grundvoraussetzungen zu erfüllen sind. Der Einbezug einer ethischen Unterstützung ist kein Ersatz für die im Alltag notwendigen ethischen Diskussionen innerhalb eines Teams, sie kann jedoch in ethisch schwierigen Situationen zusätzliche Unterstützung bieten.

\section{Ziele}

Ziel einer ethischen Unterstützung ist es, die betroffenen Personen und Institutionen bei ethisch schwierigen Werteabwägungen in ihrer Entscheidungsfindung zu unterstützen. Sie soll zur Transparenz der Entscheidungsfindung beitragen, das Erkennen von Werte- und Interessenkonflikten fördern und Lösungsansätze aufzeigen. Empfehlungen sind konsultativ: Ethische Unterstützung legitimiert sich allein durch die vorgebrachten Argumente. Die Entscheidungsverantwortung bleibt beim Arzt und dem Behandlungsteam. Auch das Recht auf Selbstbestimmung des Patienten wird dabei nicht tangiert. Es geht nicht etwa darum, Ethikstrukturen unersetzlich $\mathrm{zu}$ machen oder dem Behandlungsteam die Entschei- dungsverantwortung abzunehmen, sondern vielmehr darum, das Behandlungsteam und die Leitung von Institutionen bei ethischen Dilemmata und Konflikten mittels einer systematischen Unterstützung zu beraten. Methoden zur ethischen Reflexion können gezielt eingesetzt werden, um zur Gestaltung von Routinehandlungen, speziellen Entscheidungen sowie Strukturen und Abläufen in Spitälern, Kliniken, Heimen und bei ambulanten und gemeindenahen Dienstleistungen beizutragen. Ziele sind dabei stets,

- dem Selbstbestimmungsrecht des Patienten im ganzen Behandlungsprozess Rechnung zu tragen;

- einen Beitrag zur Entscheidungsqualität, Handlungsqualität und Reflexionstiefe zu leisten;

- die bewusste Verantwortungsübernahme und die interprofessionelle $\mathrm{Zu}$ sammenarbeit zu fördern;

- zu Transparenz und Nachvollziehbarkeit der Entscheidungen beizutragen.

In diesem Sinn sollen Ethikstrukturen einen konstruktiven Beitrag zur Sicherung der Behandlungs- und Arbeitsqualität leisten. Implizit findet ethische Reflexion in den verschiedensten Kontexten statt, die vorliegenden Empfehlungen beziehen sich aber ausschliesslich auf Strukturen, die ex- 
plizit als Orte ethischer Reflexion deklariert sind.

\section{Aufgaben}

Die ethische Unterstützung kann Folgendes umfassen: die retrospektive und prospektive Einzelfallberatung, die ethische Beratung der Leitung einer Institution, die Ausarbeitung von allgemeinen Ethikempfehlungen und -leitlinien zuhanden der Institution, die Mitwirkung bei der ethischen Aus-, Weiterund Fortbildung sowie die Forschung im Bereich Ethik und weitere Aktivitäten.

3. Grenzen, Risiken und Missverständnisse Um Missverständnisse zu vermeiden, ist es wichtig, die Ziele und das Selbstverständnis einer Ethikstruktur klar zu definieren:

- Eine ethische Unterstützung stellt keinen Ersatz für die juristische Beratung oder die Mediation in Konfliktfällen dar.

- Eine ethische Unterstützung ist nicht in allen schwierigen Situationen angebracht, sondern dann, wenn es um Wertekonflikte geht.

- Ethikstrukturen sollen die Entscheidungskompetenz der Ärzte und Pflegefachpersonen nicht einschränken, sondern diese methodisch unterstützen. Sie haben beratende Funktion und dürfen sich nicht zu einer Kontrollinstanz entwickeln.

- Eine Ethikstruktur muss unabhängig sein. Sinnvolle Arbeit kann nur geleistet werden, wenn Empfehlungen und Stellungnahmen im Rahmen medizinischer Institutionen frei geäussert werden können.

- Eine Ethikstruktur soll eine echte Hilfe für die Praxis anbieten und nicht einfach aus administrativen Gründen eingerichtet werden. Aus diesem Grund distanziert sich die SAMW von der Idee, den Aufbau von Strukturen zur ethischen Unterstützung, z.B. bei der Zertifizierung eines Spitals, zur Pflicht zu machen, wie es in den USA der Fall ist.

- Ethikstrukturen haben nicht dieselbe Funktion wie die kantonalen Ethikkommissionen, welche für die Beurteilung von Forschungsvorhaben zuständig sind und in diesem Rahmen rechtlich verbindliche Entscheide fällen.

\section{Adressaten}

Die Empfehlungen richten sich insbesondere an klinische Ethiker [5], an die Mitglieder von Ethikstrukturen sowie an alle weiteren Personen, die ethische Unterstützung anbieten und sich beratend $\mathrm{zu}$ ethischen Fragen in der Medizin äussern.

Sie richten sich ausserdem an Institutionen im stationären oder ambulanten Bereich, die, unabhängig von ihrer Grösse, ein Angebot zur ethischen Unterstützung planen oder bereits realisiert haben. Überdies richten sie sich an medizinische [6] Fachpersonen, die eine ethische Unterstützung in einem der verschiedenen Bereiche der Medizin wünschen

\section{Empfehlungen}

\section{Strukturen und Methoden}

Die Wahl der Ethikstruktur soll den ihr übertragenen Aufgaben entsprechen. Bis heute hat sich keine Struktur als den anderen überlegen ausgezeichnet, weder auf theoretischer Ebene noch in der Praxis. Wichtig ist die Entscheidung, ob die ethische Unterstützung durch eine Gruppe (Kommission oder Team), eine Einzelperson oder durch beides erfolgen soll. Eine Ethikstruktur kann zudem durch eine Organisation (z. B. ein Spital, Pflegeheim, Spitex) oder auch ein Netzwerk unterhalten werden. Sie kann auch von mehreren kleineren Häusern oder Einrichtungen gemeinsam getragen werden.

Es existieren verschiedene Methoden zur ethischen Unterstützung im Einzelfall. Zurzeit ist es nicht möglich, eine Methode besonders $\mathrm{zu}$ empfehlen, weder aufgrund der philosophischen Konzepte noch aufgrund der empirischen Daten. Die Akademie der Medizinischen Wissenschaften begrüsst es, wenn in diesem Bereich geforscht wird. Die Wahl der Methode(n) soll sich an den Rahmenbedingungen und den Aufgaben der Ethikstruktur orientieren. Diese Empfehlungen definieren lediglich diejenigen Grundvoraussetzungen, die unabhängig vom gewählten Ansatz gelten sollen.

\section{Ethische Unterstützung}

\subsection{Anfrage für eine}

\section{ethische Unterstützung}

Alle Anfragen bezüglich einer ethisch schwierigen Situation (diese kann sich z.B. durch ein Unbehagen, eine Uneinigkeit, eine Unsicherheit über die richtige Vorgehensweise äussern) fallen potentiell in den Aufgabenbereich der Ethikstruktur. Solche Anfragen können beispielsweise Themen wie Unsicherheiten in Bezug auf Behandlungsentscheide, Fragen zur gerechten Ressourcenverteilung, Pflichten der Behandelnden, Unsicherheiten in einem inter- kulturellen Kontext, Entscheidungen am Lebensende oder auch Zwangsmassnahmen betreffen. Jede in der konkreten Situation betroffene Person sollte eine ethische Unterstützung bei Einzelfallentscheiden anfordern können.

Alle Anfragen werden - unter Wahrung der Vertraulichkeit - entgegengenommen. Innerhalb der Ethikstruktur wird entschieden, wie weit die Anfrage dort bearbeitet oder an andere Instanzen wie die Ombudsstelle oder den Rechtsdienst weitergeleitet wird. Liegt der Anfrage ein Wertekonflikt zugrunde, wird sie im Rahmen der ethischen Unterstützung behandelt.

\subsection{Ethische Unterstützung bei Einzelfallentscheidungen}

Ethische Unterstützung bei Einzelfallentscheiden sollte in einem interdisziplinären Rahmen stattfinden und, so weit möglich, alle Mitglieder des Behandlungs- und Betreuungsteams mit einbeziehen. Sie kann sich an verschiedenen Methoden orientieren und die Mitglieder des Behandlungsund Betreuungsteams auf unterschiedliche Art und Weise einbeziehen. Eine Methode besteht darin, dass ein Mitglied der Ethikstruktur den Entscheidungsfindungsprozess des Behandlungsteams ethisch moderiert. Eine zweite Methode besteht darin, dass das Behandlungsteam der Ethikstruktur die Fragestellung unterbreitet und diese eine Empfehlung abgibt. Diese beiden Methoden können kombiniert werden. Wichtig ist es, die Wertvorstellungen der Teilnehmenden zu klären und auf ethische Richtlinien, relevante gesetzliche Grundlagen und die in der Literatur vertretenen Positionen hinzuweisen. Anfragen für eine ethische Unterstützung erfordern häufig eine rasche Reaktion. Die ethische Unterstützung sollte deshalb so organisiert sein, dass sie dringende Anfragen möglichst umgehend behandeln kann.

Für jede Ethikstruktur soll grundsätzlich geklärt werden, wie der Patient und dessen Angehörige in den Unterstützungsprozess einbezogen werden. Falls Mitglieder des Behandlungs- und Betreuungsteams gleichzeitig auch Mitglieder der Ethikstruktur sind, nehmen sie an der Beratung nur in der Rolle der Behandelnden teil.

In Situationen, in welchen kein Konsens entsteht, wird empfohlen, die unterschiedlichen Positionen mit den Argumenten darzulegen und darauf zu verzichten, den Eindruck einer Übereinstimmung zu erwecken (z.B. durch eine Abstimmung). 
Bei der Dokumentation müssen mindestens die Ausgangssituation, das Problem, die Teilnehmenden, der Lösungsvorschlag und dessen Begründung festgehalten werden. Falls ein Mitglied z.B. aufgrund eines Interessenkonflikts in den Ausstand getreten ist, ist dies ebenfalls anzugeben. Im Protokoll ist auch festzuhalten, wie die Weitergabe der Information und die Umsetzung erfolgen sollen; die dafür zuständige Person ist zu bezeichnen. Das Protokoll wird datiert und bei Bedarf ein Datum für die Evaluation festgesetzt. Im Patientendossier ist ferner festzuhalten, dass eine ethische Fallbesprechung stattgefunden hat, sowie die für die Behandlung und Betreuung des Patienten relevanten Aspekte. Dabei muss beachtet werden, dass der Patient das Recht auf $\mathrm{Zu}$ gang zu diesen Informationen hat.

Eine ethische Unterstützung beim Einzelfallentscheid kann sowohl prospektiv wie auch retrospektiv erfolgen. Eine ethische Fallbesprechung kann in einer Aus-, Weiteroder Fortbildung integriert sein. Allen Beteiligten sollte der angestrebte Lerneffekt bekannt sein.

\subsection{Erstellen von Ethikleitlinien}

In Ethikleitlinien werden wiederkehrende Probleme oder Wertekonflikte aufgenommen, wie beispielsweise der Umgang mit Patientenverfügungen, Reanimation, Fixation, Ernährung am Lebensende, Bluttransfusion bei Zeugen Jehovas. Sie orientieren sich an den Fragestellungen, die in Spitälern, im ambulanten Sektor oder in Einrichtungen der Langzeitpflege auftreten. Ethikleitlinien werden durch Mitglieder einer Ethikstruktur in Zusammenarbeit mit weiteren sachkundigen Personen und Mitgliedern der Leitungsebene erstellt. Bei Bedarf können zusätzliche Experten hinzugezogen werden.

Ethikleitlinien enthalten inhaltliche Aspekte, eine ausgearbeitete ethische Begründung sowie eine explizite Wertereflexion. Dabei berücksichtigen sie die spezifischen Herausforderungen der betroffenen Institution. Sie müssen den gesetzlichen Vorschriften, den medizin-ethischen Richtlinien der SAMW sowie dem wissenschaftlichen Standard entsprechen. Nach Erarbeitung einer vorläufigen Fassung der Leitlinie soll diese den am meisten involvierten $\mathrm{Ab}$ teilungen und Anwendern zur Beurteilung vorgelegt werden.

Sollen sie verbindlichen Charakter haben, müssen Ethikleitlinien von der zuständigen Leitung einer Einrichtung in Kraft gesetzt werden. Ihre Gültigkeit und Verbindlichkeit für die Institution muss klar deklariert werden. Ethikleitlinien sollten in Einführungsveranstaltungen, in internen Informationsveranstaltungen, Aus-, Weiterund Fortbildungen und/oder mittels interner Publikationen (Zeitschriften, Intranet usw.) vorgestellt werden.

Ethikleitlinien müssen in regelmässigen Abständen auf ihre praktische Anwendbarkeit und auf ihre Aktualität hin überprüft und gegebenenfalls angepasst werden.

\subsection{Aus-, Weiter- und Fortbildung}

Ethikstrukturen können einen wichtigen Beitrag zur Aus-, Weiter- und Fortbildung leisten, da ihre Mitglieder die Schwierigkeiten und Abläufe in der Institution besonders gut kennen.

Die kontinuierliche Aus-, Weiter- und Fortbildung der Mitglieder von Ethikstrukturen ist sicherzustellen. Für die Mitarbeitenden einer Institution angebotene Aus-, Weiterund Fortbildungsanlässe sollten sowohl theoretische Kenntnisse als auch praktische Fähigkeiten vermitteln.

Mitglieder der Ethikstruktur können zudem durch die Teilnahme an Debatten und Vorträgen zur öffentlichen Weiterbildung in ethischen Fragestellungen beitragen.

\section{Aufbau und Unterhalt von Strukturen zur ethischen Unterstützung (Ethikstrukturen)}

\subsection{Strukturelle Voraussetzungen und Unabhängigkeit}

Ethikstrukturen müssen in der Trägerorganisation sichtbar verankert sein. Die Verantwortlichen unterstützen sie und stellen ihnen die notwendigen Ressourcen zur Verfügung. Die zur Verfügung gestellten personellen und materiellen Ressourcen (z.B. Sekretariat oder Mittel für die Ausbildung der Mitglieder einer Ethikstruktur) entsprechen den ihnen gestellten Aufgaben. Dies setzt voraus, dass die Mitglieder sich während der Arbeitszeit im erforderlichen Mass in der Ethikstruktur engagieren können.

Profil und Auftrag der Ethikstruktur müssen klar definiert sein. Überschneidungen mit anderen Stellen, die ähnliche Fragen bearbeiten, wie z.B. Rechtsdienst und Mediation, müssen definiert sein.

Das Angebot einer ethischen Unterstützung setzt eine Betriebskultur voraus, die offen ist für eine Auseinandersetzung mit möglicherweise kontroversen Fragestellungen, für mögliche ethische Konflikte, für interdisziplinäre Auseinandersetzungen und auch für die Möglichkeit, praktische Konsequenzen daraus abzuleiten. Es braucht ausserdem einen genügend grossen Kreis von Mitarbeitenden, der sich im Bereich der Ethik engagieren will und kann. Das hierfür notwendige Interesse kann auch durch vorgängige Aus-, Weiter- und Fortbildungen gefördert werden.

Die Ethikstruktur muss «zugänglich» sein. Vorgesetzte schaffen die Rahmenbedingungen dafür, dass eine ethische Unterstützung tatsächlich in Anspruch genommen werden kann, indem sie z. B. Zeit zur Verfügung stellen. Die Inanspruchnahme einer ethischen Unterstützung soll jedoch jederzeit unabhängig vom Einverständnis der Vorgesetzten möglich sein. Vorgesetzte sollen darin bestärkt werden, dies nicht als Bedrohung, sondern als Hilfestellung zu begreifen.

Die Nützlichkeit einer Ethikstruktur gründet gleichzeitig in ihrer Unabhängigkeit in der Funktion und in ihrer organisatorischen Anbindung an die Institution. Die Ethikstruktur sollte so unabhängig wie möglich funktionieren; Abhängigkeiten (inkl. diejenigen, die auf die Sozialisierung innerhalb der Institution zurückzuführen sind) müssen reflektiert werden. Ein transparenter Umgang mit Abhängigkeiten, eingeschliffenen Handlungsmustern und Interessenkonflikten aller Art kann vor Instrumentalisierung schützen. Handeln aus Eigeninitiative muss möglich sein. Teilzeitanstellungen, Vernetzungen, die Präzisierung der Unabhängigkeit in Verträgen usw. können die Unabhängigkeit fördern. Das Reglement muss einen genügenden Grad an Unabhängigkeit garantieren.

\subsection{Etablierung von Ethikstrukturen}

Die Etablierung einer Ethikstruktur innerhalb einer Trägerorganisation erfordert eine optimale Koordination zwischen Initiativen der Basis (bottom up) und der Leitung der Organisation (top down). Ein einseitiger Ansatz hätte wenig Erfolgschancen: Die Nachfrage und der Bedarf nach ethischer Unterstützung bleiben unbefriedigt, wenn bei der Leitung der Trägerorganisation die Sensibilität für die Bedeutung ethischer Fragen nicht vorhanden ist. Auf der anderen Seite besteht auch das Angebot zur Unterstützung nur formal, wenn dieses nicht in Anspruch genommen wird.

Anfänglich sollte eine kleine interdisziplinäre Gruppe einen Vorschlag für eine Institution, die eine Ethikstruktur einrichten 
möchte, ausarbeiten. Im Idealfall setzt sich diese Gruppe aus Personen zusammen, die aus allen Hierarchiestufen stammen. Mindestens eine Person sollte über besondere fachliche Kompetenz im Bereich Ethik verfügen. Die Gruppe einigt sich hinsichtlich ihrer Definition von «ethischer Unterstützung», sie verschafft sich einen Überblick über die bekannten Methoden und arbeitet ein Konzept und ein Reglement aus. Dieses beschreibt den Auftrag und die Funktionsweise der Ethikstruktur. Eine anfängliche Sensibilisierung, z.B. durch Fortbildungen oder Symposien, ist oft hilfreich. Es ist zudem wichtig, dass die Aufgaben und Grenzen einer Ethikstruktur von Anfang an geklärt werden.

\subsection{Zusammensetzung}

Handelt es sich um ein Gremium von mehreren Personen, soll grosses Gewicht auf eine vielfältige Zusammensetzung gelegt werden. Dabei wird mindestens folgende Zusammensetzung empfohlen:

- Vertreter aus verschiedenen Fachbereichen und Berufsgruppen der Medizin;

- Personen, die innerhalb der Institution in unterschiedlichen Hierarchiestufen tätig sind;

- $\quad$ ein Ethiker [7] oder eine andere Fachperson, die über eine vertiefte Ausbildung im Bereich der biomedizinischen bzw. klinischen Ethik verfügt. Der Ethiker kann auch in einem Auftragsverhältnis stehen.

Zusätzlich werden Vertreter aus folgenden Bereichen empfohlen:

- Personen, die im Bereich der Medizin tätig sind, jedoch ausserhalb der Institution arbeiten;

- ein Jurist, Psychologe, Sozialarbeiter, Seelsorger usw.;

- Personen, die ausserhalb der Institution stehen [8].

\subsection{Grundeinstellungen, Kenntnisse und Kompetenzen}

Die nachfolgend aufgeführten Anforderungen sollten in jeder Ethikstruktur erfüllt sein, unabhängig davon, ob es um ein Gremium oder Einzelpersonen geht. Handelt es sich um ein Gremium, können spezielle Anforderungen von einem einzelnen oder mehreren Mitgliedern erfüllt werden.

Die Mitglieder einer Ethikstruktur bringen bestimmte Grundeinstellungen mit:

- Sie sind bereit, die eigenen Wertvorstellungen zu reflektieren;
- Sie sind fähig, eigene Überzeugungen zu formulieren und zu vertreten;

- Sie sind bereit, aufgrund der gemeinsamen Diskussion eigene Ansichten zu überdenken;

- Sie sind bereit, sich aktiv zu engagieren;

- Sie sind offen für Gespräche und für Argumente, die von anderer Seite eingebracht werden;

- Sie sind offen für andere Disziplinen und Professionen;

- Sie sind bereit, ein realistisches Verständnis des klinischen Alltags zu erwerben;

- Sie sind bereit, sich im Bereich der Ethik fortzubilden.

Die Mitglieder einer Ethikstruktur erwerben innert nützlicher Frist folgende Kenntnisse und Kompetenzen:

- Sie können die ethische Reflexion von moralischen Positionen unterscheiden;

- Sie sind vertraut mit den unterschiedlichen ethischen Theorien und können deren Argumente auf Einzelfälle beziehen;

- Sie können in einer konkreten Situation die relevanten Werte und Normen von den wichtigen Fakten unterscheiden bzw. sind vertraut mit der Unterscheidung zwischen normativen und deskriptiven Urteilen;

- Sie können zwischen Ethik und Recht unterscheiden und den wechselseitigen Bezug herstellen;

- Sie kennen die zentralen Fragestellungen der Medizinethik;

- Sie kennen die wichtigsten Quellen der ärztlichen (SAMW-Richtlinien, EthikCode der WMA) resp. pflegerischen Berufsethik (SBK-, ICN-Kodex);

- Sie kennen die Organisationen im Bereich der Standesethik (z.B. SAMW, NEK, FMH, SBK).

Jede der folgenden Kompetenzen sollte von mindestens einer Person mitgebracht werden [9]:

- Kenntnis der verschiedenen Möglichkeiten der Strukturierung ethischer Reflexion in Fallsituationen und anderen konkreten Fragen;

- Fähigkeit zur Moderation eines ethischen Entscheidungsprozesses;

- Erkennen der moralischen Perspektiven aller Involvierten;

- Vertrautheit mit dem ethischen Argumentieren;
- Kenntnis der wesentlichen Etappen der Geschichte der Medizinethik;

- Vertrautheit mit den früheren und aktuellen Debatten über relevante bioethische Fragestellungen;

- Vertieftes medizinisches Fachwissen und Fähigkeit, dieses Wissen in einer für medizinische Laien verständlichen Art zu vermitteln;

- Pflege des Kontakts zur akademischen Forschung im Bereich der Ethik, beispielsweise mittels einer Anbindung an ein universitäres Institut für Ethik;

- vertiefte Kenntnisse im Gesundheitsrecht.

Je nach Aufgabenbereich einer Ethikstruktur kann Fachwissen in weiteren Bereichen, z. B. der Organisationstheorie, Interkulturalität, Soziologie, Psychologie oder Seelsorge, von Nutzen sein. Dieses spezifische Fachwissen kann auch in Form von Expertisen eingeholt werden.

Es gibt verschiedene Formen, sich diese Kenntnisse und Kompetenzen anzueignen, namentlich Teilnahme an einer von der Ethikstruktur organisierten Aus-, Weiteroder Fortbildung. Die Modalitäten sollten in einem Reglement festgehalten werden. Dieses hält auch fest, in welcher Form der Nachweis über die absolvierte Aus-, Weiter- und Fortbildung zu erbringen ist. Die in einer Ethikstruktur engagierten Personen sollen die Gelegenheit haben, sich während der Arbeitszeit aus-, weiter- und fortzubilden.

\subsection{Sichtbarkeit der Ethikstruktur}

Es ist wichtig, dass die Ethikstruktur über eine geeignete Kommunikationsstrategie verfügt, um sich bekannt zu machen und die Zugänglichkeit zu erhöhen. Die dafür zur Verfügung stehenden Mittel variieren je nach Institution (z.B. hausinterne Zeitung, Inter- und/oder Intranet, Flyer für Mitarbeitende und Patienten, Plakate). Damit die ethische Unterstützung niederschwellig angefragt werden kann, soll eine Kontaktmöglichkeit bezeichnet werden.

\subsection{Praktische Hinweise}

Ethikstrukturen sind mit drei Arten von Fragestellungen konfrontiert:

- Welche Struktur für die ethische Unterstützung im Einzelfall soll gewählt werden?

- Wie soll die ethische Unterstützung organisiert sein?

- Welche Diskussionsmethoden bieten sich an? [10] 


\subsubsection{Welche Struktur für}

ethische Unterstützung im Einzelfall?

Ethische Unterstützung kann geleistet werden durch eine Kommission, ein kleines Team oder durch einen Ethikberater.

- Eine Kommission hat den Vorteil, dass viele verschiedene Sichtweisen und eine spezifische Kenntnis in Ethik immer vorhanden sind, aber es ist für sie schwieriger, in dringlichen Fällen innert angemessener Frist zu reagieren.

- Ein Ethikberater verfügt über eine spezifische Kompetenz in Ethik, steht aber vor der Herausforderung, die gesamte Bandbreite der Argumente abdecken zu müssen.

- Die Beratung durch ein kleines Team lässt eine grössere Flexibilität zu als die Beratung durch die Gesamtkommission oder eine Einzelperson.

Kombinationen aus diesen verschiedenen Strukturen sind ebenfalls möglich.

\subsubsection{Wie soll die ethische Unterstützung organisiert sein?}

Folgende konkrete Fragen sind zu beantworten:

- Die Art und Weise, wie Patienten und Mitarbeitende über die Existenz einer Ethikstruktur informiert werden.

- Die Voraussetzungen für die Anfrage an die Ethikstruktur.

- Das Vorgehen bei einer Anfrage für eine ethische Unterstützung im Einzelfall.

- Das Vorgehen, wenn ein einzelnes Mitglied der Ethikstruktur informell um eine Einschätzung zu einer ethischen Frage gebeten wird.

- Das Vorgehen in einer Notfallsituation, in welcher eine rasche Rückmeldung erforderlich ist, und die minimale Frist, in welcher eine Stellungnahme möglich ist.

- Die Zusammensetzung des Gremiums, welches eine ethische Unterstützung anbietet, oder wenn die Zusammensetzung fallweise erfolgt, nach welchen Kriterien dies im Einzelfall geschieht. Dabei sollte auch geklärt werden, welchen Platz Personen haben, die keine ethische Unterstützung angefordert haben: z. B. der Patient, wenn das Behandlungsteam die ethische Unterstützung verlangt hat, oder umgekehrt das Behandlungsteam, wenn der Patient oder seine Angehörigen die Unterstützung verlangen.

- Die Art und Weise, wie das Gremium über Stellungnahmen informiert.
- Die Art und Weise, wie das Ergebnis der ethischen Unterstützung festgehalten und archiviert wird.

- Die Art und Weise, wie die Vertraulichkeit gewährleistet wird.

Für jede Ethikstruktur muss auch eine Anzahl organisatorischer Fragen geklärt werden [11]:

- wie gewährleistet wird, dass bei jeder Ethikberatung eine genügende Fachkompetenz im Bereich Ethik und in den spezifisch medizinisch-pflegerischen und institutionellen Fragen besteht.

- ob es eine Amtszeitbegrenzung respektive eine Mindestdauer für die Mitgliedschaft in einer Ethikstruktur gibt.

- wie und nach welchen Kriterien neue Mitglieder rekrutiert werden.

- wie die administrative Unterstützung sichergestellt wird. Welches Budget dafür vorhanden ist.

- welche Mittel für die Aus-, Weiter- und Fortbildung der Mitglieder zur Verfügung stehen.

- und falls das Engagement von Mitgliedern nicht in die Arbeitszeit fällt, oder externe Personen der Ethikstruktur angehören, wie diese entschädigt werden.

Bei der Etablierung einer Ethikstruktur sollten diese Fragen geklärt und die wesentlichen Punkte in einem Reglement festgehalten werden.

\subsection{Anforderungen an Ethikstrukturen für spezifische Bereiche}

Die bestehenden Methoden und Strukturen zur ethischen Unterstützung wurden vor allem für die Bedürfnisse von Akutspitälern entwickelt. Die ethische Unterstützung muss entsprechend den Bedürfnissen verschiedener Bereiche angepasst werden. Spezifische Anpassungen werden hier vorgeschlagen für die Akutversorgung in einem grossen Spital, die Psychiatrie, Pädiatrie, Langzeitpflege, ambulante und gemeindenahe Versorgung und die Gefängnismedizin sowie für die ethische Unterstützung der Spitalleitung und der Verwaltung von Gesundheitsinstitutionen.

\subsubsection{Akutversorgung in grösseren Spitälern}

Die ersten Ethikstrukturen sind im Bereich Akutversorgung entstanden und die meisten Modelle sind für diesen Kontext entwickelt worden. Dennoch ist es wichtig, die spezifischen Merkmale im Vergleich zu anderen Bereichen der Gesundheitsversorgung festzuhalten, namentlich:
- Die Beziehungen zwischen den Mitarbeitenden in einer grösseren Institution sind oft weniger direkt und klar definiert als in einem kleineren Spital. In Abhängigkeit von der Grösse der Institution sind unterschiedliche Ansätze und Massnahmen nötig, um die Ethikstruktur bekannt zu machen, Vertrauen zu schaffen und die Unterstützung der Leitung der Institution - formell und informell - zu gewinnen.

- Die Art der Fragestellungen, die der Ethikstruktur unterbreitet werden, ist sehr vielfältig. Aus diesem Grund sind die Mitglieder der Ethikstruktur häufiger mit Situationen konfrontiert, die ausserhalb ihrer alltäglichen Erfahrung liegen.

- Die möglicherweise höhere Fluktuation des Personals kann in einem Spannungsverhältnis zu der minimal notwendigen Stabilität einer Ethikstruktur stehen.

- Der Aufenthalt eines Patienten in einem grossen Krankenhaus ist häufig durch Verlegungen und Wechsel des Behandlungsteams gekennzeichnet, was einerseits ethische Fragestellungen aufwerfen und andererseits die Arbeit der Ethikstruktur komplexer gestalten kann.

- Es können mehrere voneinander unabhängig arbeitende Ethikstrukturen nebeneinander bestehen.

- Ein universitäres Institut kann ebenfalls im Bereich der medizinischen Ethik tätig sein.

Aus diesen Merkmalen ergeben sich folgende Punkte, die eine Ethikstruktur berücksichtigen sollte:

- Um die Ethikstruktur bekannt zu machen, sollte eine systematische Informationsstrategie gewählt werden.

- Ein regelmässiger Wechsel der Mitglieder der Ethikstruktur kann dazu beitragen, die Bekanntheit der Struktur und die ethische Sensibilität innerhalb der Organisation zu erhöhen, da dies dazu führt, dass ehemalige Mitarbeiter in verschiedenen Abteilungen arbeiten.

- Es ist unerlässlich, dass die Mitglieder der Ethikstruktur verschiedene Berufsgruppen und medizinische Disziplinen vertreten, damit die klinische Erfahrung möglichst breit abgestützt ist.

- Es wird empfohlen, bei der Auswahl der Mitglieder diejenigen zu bevorzugen, die über längere Zeit in der Institution arbeiten.

- Aufgrund der hohen Personalfluktuation sollten Bemühungen, die Mitarbei- 
tenden für ethische Fragen zu sensibilisieren, wiederholt stattfinden.

- Das Vorhandensein mehrerer unabhängig voneinander bestehender Ethikstrukturen sollte durch spezifische Bedürfnisse begründet und die jeweilige Rolle geklärt werden. Die Koordination und Zusammenarbeit zwischen den verschiedenen Strukturen ist anzustreben.

- An Universitätsspitälern ist die Zusammenarbeit mit dem zuständigen universitären Institut für medizinische Ethik anzustreben.

\subsubsection{Psychiatrie}

Patienten mit einer psychiatrischen Erkrankung werden sowohl stationär als auch ambulant betreut. Die Probleme, mit denen die Ethikstruktur konfrontiert ist, betreffen deshalb Themen mit einer grossen Bandbreite (Anwendung von Patientenverfügungen, Ablehnung einer Behandlung, Alternativen zur Zwangsbehandlung, Wunsch nach Beihilfe zum Suizid usw.).

Folgende Aspekte sind für die Psychiatrie spezifisch:

- Die psychiatrische Erkrankung kann einen teils schwankenden, teils dauerhaften Einfluss auf die Urteilsfähigkeit haben. Die Beurteilung der Urteilsfähigkeit ist Inhalt der Therapie.

- Relativ häufig stellt sich die Frage der Gefährlichkeit des Patienten für sich selbst, für seine Umgebung sowie für die Pflegenden.

- Das Umfeld des Patienten, wie z. B. informelle Pflegepersonen (Familie), Vormund, Pflegebeistand, oft Pflegepartner, spielt eine wesentliche Rolle.

- Die ausgeprägte Individualisierung der Behandlung eignet sich weniger für den Gebrauch von standardisierten Behandlungsprotokollen für eine Krankheit.

Daraus ergeben sich folgende Punkte, die eine Ethikstruktur berücksichtigen sollte:

- Teilweise sind spezielle Anstrengungen erforderlich, damit Patienten mit einer psychiatrischen Erkrankung in der ethischen Unterstützung nicht anders behandelt werden als Patienten aus anderen Bereichen (z.B. der Einbezug des Patienten und - mit seinem Einverständnis - seiner Angehörigen).

- Empfehlungen, die für andere Bereiche entwickelt wurden, müssen oft an die spezifischen Bedürfnisse der Psychiatrie angepasst werden. So sind beispielsweise
Patientenverfügungen häufiger geworden in der Psychiatrie. Das Verfassen einer Patientenverfügung, teilweise mit Unterstützung der medizinischen Betreuungspersonen im ambulanten Bereich oder am Ende einer Hospitalisation, erlaubt es dem Patienten, therapeutische Präferenzen für eine nächste Krisensituation festzuhalten und eine spezifische Übereinkunft zu treffen.

- Die Einsicht in ihr Patientendossier wird von Psychiatriepatienten häufiger gefordert. Bei der Dokumentation der Beratung muss - wie üblich - die ärztliche Geheimhaltungspflicht betreffend Informationen, die von Drittpersonen stammen, respektiert werden.

Die Unterstützung durch eine Ethikstruktur kann eine Chance sein, den Dialog zwischen dem Patienten und den Therapeuten zu erneuern und die Grundlagen der Behandlung besser an die Bedürfnisse des Patienten anzupassen. Sie muss von einem Rekurs gegen freiheitsbeschränkende Massnahmen unterschieden werden.

\subsubsection{Pädiatrie und Neonatologie}

In der Pädiatrie und insbesondere in der Neonatologie wurde das Bedürfnis nach einer strukturierten Unterstützung bei der Behandlung ethischer Fragen schon relativ früh akut manifest und hat in der Schweiz zur Ausarbeitung verschiedener Modelle geführt. Besonderheiten im Vergleich zur Akutmedizin für Erwachsene sind:

- ausserordentlich langfristige Auswirkungen akut dringlicher Behandlungsentscheidungen (wie z. B. betreffend Intensivtherapie von extrem Frühgeborenen);

- hohe prognostische Unsicherheit bezüglich Mortalität und Langzeitmorbidität;

- bei Neugeborenen, Säuglingen und Kleinkindern fehlende Anhaltspunkte für einen mutmasslichen Willen;

- die Urteilsfähigkeit des Patienten entwickelt sich im Lauf der Zeit;

- Eltern sind gesetzliche Vertreter und gleichzeitig von allen Entscheidungen auch massiv und langfristig persönlich Betroffene.

Aufgrund dieser Besonderheiten und der starken Tradition des Arbeitens in multiprofessionellen Behandlungsteams sollten Ethikstrukturen in der Pädiatrie und Neonatologie folgende Punkte berücksichtigen:

- Vertrautheit einer genügenden Anzahl der an einer Ethikstruktur beteiligten
Personen mit den besonderen Gegebenheiten der Pädiatrie ist essentiell;

- Eine mindestens partielle Eigenständigkeit gegenüber den Ethikstrukturen der Erwachsenenmedizin ist von Vorteil, auch wenn die Pädiatrie Teil eines Allgemeinspitals ist;

- Die Art, wie Eltern in therapeutische Entscheide eimbezogen werden, sollte in jeder Institution grundsätzlich geklärt werden;

- Mindestens eine Person sollte mit den Grundzügen des Kinderschutzes gut vertraut sein. Die Schnittstelle zu den an allen Kinderspitälern existierenden Kinderschutzgruppen muss geklärt werden.

\subsubsection{Langzeitpflege}

Institutionen der Langzeitpflege sind in der Regel gleichzeitig auch Wohnsitz der dort betreuten Menschen. Medizinische und pflegerische Entscheidungsprozesse mit ethischer Relevanz in der Langzeitpflege treten täglich auf und betreffen eine äusserst vulnerable Gruppe von Menschen. Für die Langzeitpflege sind folgende Aspekte spezifisch:

- Die Patienten sind aus physischen, psychischen und/oder psychosozialen Gründen in hohem Masse von anderen abhängig und auf Schutz und Unterstützung angewiesen.

- Die Patienten sind häufig in ihrer Autonomiefähigkeit eingeschränkt resp. nur in bestimmten Belangen urteilsfähig, oder ihre Urteilsfähigkeit ist schwierig festzustellen.

- Die Patienten haben aus medizinischen Gründen oft nicht die Kraft oder Möglichkeit, ihren Willen ohne fremde Unterstützung auszudrücken oder durchzusetzen.

- Das Zusammenleben unterschiedlichster pflegebedürftiger Menschen und die wechselseitige Beziehung zwischen Patienten, Betreuenden und Familie können zu Spannungen und auch zu Gewalt führen.

- An einem Ort, wo sich vieles in einem Gemeinschaftsbereich abspielt, ist es schwierig, die Privatsphäre zu bewahren.

- Die Gefahr der Über- oder Unterbehandlung ist gross bei Menschen, die in einer Pflegeinstitution leben, insbesondere, wenn sie an einer Demenz oder an einer anderen gerontopsychiatrischen Krankheit leiden. 
- In Pflegeinstitutionen arbeiten viele Mitarbeitende ohne spezifische fachliche Ausbildung, oft auch Mitarbeitende aus anderen Kulturen.

- Durch langdauernde und umfassende Pflege und Betreuung entstehen persönliche Beziehungen zwischen Personal und Patienten, welche die Entstehung, Wahrnehmung und Lösung ethischer Probleme positiv oder negativ beeinflussen können.

Sensibilisierung und das Vorhandensein von ethischen Kompetenzen sind deshalb in der Langzeitpflege für alle Betreuenden (Ärzte, Pflegende, weitere Therapeuten, Sozialdienste, Seelsorge), die immer wieder alltagsethische Entscheide treffen müssen, unerlässlich.

Für übergeordnete ethische Fragestellungen, wie ethische Richtlinien zur Anwendung von freiheitsbeschränkenden Massnahmen oder zum Umgang mit Beihilfe zum Suizid, oder die Bearbeitung von Entscheidungsgrundlagen, ist eine Ethikstruktur geeignet, welche aus den verschiedenen Berufsgruppen und Hierarchiestufen zusammengesetzt ist, inklusive einer Vertretung der Institutionsleitung und der Trägerschaft. Mehrere Institutionen können sich für die Schaffung einer Ethikstruktur zusammenschliessen.

\subsubsection{Ambulanter Bereich, Spitex und gemeindenahe Versorgung}

Schwierige ethische Entscheidungssituationen sind im ambulanten Bereich nicht seltener als im Spital oder in der Langzeitpflege. Dennoch fehlen heute explizite Ethikstrukturen weitgehend. Ethische Fragestellungen werden aber oft in Balint-Gruppen oder in Qualitätszirkeln von Hausärzten diskutiert.

Die Situationen, in denen ethische Schwierigkeiten auftreten, unterscheiden sich stark. Sie schliessen sowohl Differenzen zwischen den verschiedenen Fachpersonen, die in die medizinische Betreuung eines Patienten involviert sind, als auch Fragen nach den Grenzen der medizinischen Betreuung ein. Es handelt sich um Patienten, die besonders vulnerabel und abhängig sind, teilweise sind sie nicht mehr urteilsfähig oder weisen Verhaltensauffälligkeiten auf. Bestimmte Situationen sind besonders heikel, z.B. bei Personen, die übermässig Alkohol konsumieren oder die gewalttätig sind, oder Menschen, die sich vernachlässigen, insbesondere auch, wenn sie abhängig sind und allein leben.

Falls sich im ambulanten Bereich Ethik- strukturen etablieren wollen, müssen folgende Aspekte berücksichtigt werden: - Die Pflege und Betreuung des Patienten findet in seiner privaten Wohnung statt. Persönliche Alltagsnormen unterscheiden sich häufig von professionellen Normvorstellungen. Betreuungsprobleme mit ethischen Dimensionen tangieren oft auch die Struktur des Alltags des Patienten und/oder seiner Angehörigen.

- Die Beziehung zwischen Patient und den medizinischen Betreuungspersonen ist häufig persönlich. Dies kann ethische Entscheidungsfindungen vereinfachen oder die Erkennung von Problemen behindern.

- Die Qualität sowie die Kontinuität der Betreuung im ambulanten Bereich hängen häufig von den Angehörigen eines Patienten ab. Die Kontakte mit dem betreuenden Arzt, weiteren Ärzten und Fachpersonen sowie der Spitex und anderen Anbietern werden ebenfalls häufig von den Angehörigen wahrgenommen.

- Unklare Verantwortlichkeiten zwischen Patient, Angehörigen und medizinischen Fachpersonen können das Entstehen von ethisch schwierigen Situationen fördern.

- Die im ambulanten Bereich direktere Konfrontation mit den finanziellen Problemen kann zu Spannungen zwischen Patienten und betreuenden Medizinalpersonen führen.

- In einer Notfallsituation ist es schwierig, eine ethische Unterstützung anzubieten. Die Anzahl involvierter medizinischer Fachpersonen und ihr Wechsel können zudem zu einer Fragmentierung der Versorgung führen. Deshalb ist es schwierig, ein Treffen zwischen Fachpersonen und Angehörigen zu organisieren. Dies kann einerseits ethische Schwierigkeiten hervorrufen, andererseits die Arbeit der Ethikstruktur erschweren.

Aus diesen Merkmalen ergeben sich folgende Punkte, die bei der Einrichtung einer Ethikstruktur berücksichtigt werden sollten:

- Für Fachpersonen im ambulanten Bereich (Spitex, Hausärzte) sollte ein Angebot für eine Aus-, Weiter- und Fortbildung im Bereich Ethik bestehen.

- Die Ethikstruktur soll im Idealfall für dringende Fälle einfach und rasch (z.B. per Telefon) zugänglich sein.

- Sie sollte ausserdem die Teilnahme an interdisziplinären Treffen im Rahmen der komplexeren Fälle ermöglichen.
- Soweit sie Verantwortung für die Betreuung eines Patienten tragen, müssen die Angehörigen in die ethischen Überlegungen einbezogen werden.

- Wenn mehrere Institutionen bei der klinischen Betreuung mitwirken, die je eine Ethikstruktur haben, ist die ethische Diskussion zu koordinieren.

\subsubsection{Gefängnismedizin}

In der Gefängnismedizin stellen sich spezifische Probleme [12]. Für Ethikstrukturen in der Gefängnismedizin sind folgende Aspekte relevant:

- Es ist schwierig, im Gefängnis eine medizinische Behandlung und Betreuung anzubieten, die jener der Zivilbevölkerung entspricht.

- Es gibt Situationen, in denen Aufsichtspersonal involviert ist, obwohl es weder zum medizinischen Behandlungsteam noch zu den Angehörigen des Patienten im Strafvollzug gehört.

- Die Unabhängigkeit der Ethikstruktur muss demnach nicht nur gegenüber dem Spital oder Ambulatorium, sondern auch gegenüber der Gefängnisverwaltung gewährleistet werden.

Bei der Einrichtung von Ethikstrukturen sollten folgende Punkte beachtet werden:

- Die institutionelle Anbindung der Ethikstruktur muss möglichst im Gesundheitswesen erfolgen. Die Unabhängigkeit einer ad hoc zur Verfügung gestellten Ethikstruktur in einem Gefängnis ist schwieriger zu gewährleisten.

- Die Mitglieder der Ethikstruktur sollten sich vor Ort treffen können.

- Das medizinische Personal des Gefängnisses muss in den Entscheidungsprozess der Ethikstruktur eingeschlossen werden. Es muss sichergestellt werden, dass das notwendige Wissen über die medizinische Versorgung im Gefängnis vorhanden ist.

- Es muss ein Vorgehen definiert werden für Entscheidungen darüber, in welchen Situationen und wie ein oder mehrere Mitarbeitende(r) des Gefängnisses in den Entscheidungsprozess einbezogen werden können.

\subsubsection{Institutionsleitung und Verwaltung}

Spitalleitung und Verwaltung sollten ebenfalls auf die Unterstützung durch eine Ethikstruktur zurückgreifen können.

Eine solche Beteiligung zur Unterstützung der Führungsebene von Institutionen 
birgt allerdings Interessenkonflikte zwischen ökonomischen, institutionellen und politischen Faktoren und Abhängigkeiten, die sorgfältig geregelt werden müssen. So sollte die Ethikstruktur beispielsweise die Möglichkeit haben, Aufträge abzulehnen, ohne dass dies negative Konsequenzen hat, insbesondere wenn es sich um Fragen der Wirtschafts- oder Umweltethik handelt. Aus diesem Grund ist es von Vorteil, wenn die ethische Unterstützung der Führungsebene durch ein Gremium erfolgt, z.B. durch die Ethikkommission im Haus und nicht durch eine Einzelperson. In gewissen Situationen kann es sinnvoll sein, extern eine ethische Unterstützung einzuholen, um eine grössere Unabhängigkeit zu gewährleisten.

Innerhalb von Ethikstrukturen, welche die Spitalleitung unterstützen, sollen betriebswirtschaftliche und juristische Grundkenntnisse verfügbar und eine gewisse Vertrautheit mit der nationalen Gesundheitspolitik und deren aktuellen Entwicklungen vorhanden sein.

\section{Qualität}

Die Qualität der ethischen Unterstützung soll fortlaufend reflektiert werden. Die Ethikstruktur soll dazu über entsprechende Mittel verfügen. Dabei sollte bewusst bleiben, dass die Evaluation der Qualität ein schwieriges Unterfangen bleibt; quantitative Messmethoden, die in anderen Bereichen zum Einsatz kommen, können dabei nicht einfach übernommen werden. Hier besteht Forschungsbedarf, und die Schweizerische Akademie der Medizinischen Wissenschaften würde es begrüssen, wenn diese Fragen untersucht würden.

Für die Überprüfung der Qualität der ethischen Unterstützung sollten Ziele formuliert und überprüft werden, inwieweit diese erreicht werden. Die Wahl dafür geeigneter Kriterien bedarf allerdings vertiefter Reflexion und Erprobung. Die Anzahl von Anfragen an die Ethikstruktur ist z. B. nicht zwingend ein guter Indikator für Qualität. Eine hohe Anzahl von Anfragen kann beispielsweise bedeuten, dass der Zugang zur Ethikstruktur einfach ist, oder auch, dass die Fallbesprechungen wenig Lerneffekt haben.

Aus diesem Grund sollte der Austausch von Erfahrung und Wissen zwischen Ethikstrukturen gefördert werden; dies könnte beispielsweise auch durch ein Peer-Reviewsystem erfolgen. Auch eine Rückmeldung der Personen, welche die Ethikstruktur angefragt haben, kann hilfreich sein.
Wenn innerhalb einer Ethikstruktur schwerwiegende Probleme auftreten, muss dies korrigiert werden, ohne ihre Unabhängigkeit zu gefährden.

Vom Senat der SAMW

am 29. Mai 2012 genehmigt.

Die französische Fassung ist die Stammversion.

\section{Anmerkungen}

1 Es gibt keinen in der deutschen Literatur etablierten Begriff, der die explizite Entwicklung und Förderung ethischer Kenntnisse, Fähigkeiten und Haltungen in der Praxis von Institutionen des Gesundheitswesens umfassend beschreibt. Nachfolgend wird in Anlehnung an das im Englischen verwendete «ethics support» von ethischer Unterstützung gesprochen. Gemeint sind alle Formen der Unterstützung: Ethikberatung, Ethikkonsil usw.

2 Aus Gründen der leichteren Lesbarkeit gilt in diesem Text die männliche Bezeichnung für beide Geschlechter.

3 Salathé M, Amstad H, Jünger M, Leuthold M, Regamey C. Institutionalisierung der Ethikberatung an Akutspitälern, psychiatrischen Kliniken, Pflegeheimen und Einrichtungen der Rehabilitation der Schweiz: Zweite Umfrage der Schweizerischen Akademie der Medizinischen Wissenschaften. Bioethica Forum. 2008;1(1):8-14.

4 Empfehlungen der SAMW haben eine geringere Verbindlichkeit als die Richtlinien der SAMW, die durch Aufnahme in die Standesordnung der FMH zu Standesrecht werden.

5 «Die Begriffe Ethiker, klinischer Ethiker, beratender Ethiker sowie Bioethiker bezeichnen einen Spezialisten der biomedizinischen Ethik, d. h. Personen mit einem geistes- oder sozialwissenschaftlichen, biomedizinischen oder gesundheitswissenschaftlichen Hintergrund, die sich im Bereich der Theorien und Methoden der biomedizinischen Ethik weitergebildet haben. Fachethiker wirken einerseits beratend bei der Lösung schwieriger klinischer Fälle, andererseits vermitteln sie Gesundheitsfachpersonen bioethisches Grundwissen.» (cf. Hottois G, Missa JN. Nouvelle Encyclopédie de bioéthique. Bruxelles: De Boeck Université. 2001).

6 Der Begriff «medizinisch» wird nachfolgend umfassend verwendet und bezieht sich au die Tätigkeit von Ärzten, Pflegenden und allen weiteren therapeutisch Tätigen im Gesundheitsbereich.

7 Vgl. Fussnote 5.

8 Bei den Personen, die ausserhalb der Institution stehen, kann es sich um Patienten, Patientenvertreter, Angehörige oder ganz generell Personen, die sich in einer Ethikstruktur engagieren wollen, handeln.
9 Diese Anforderungen stellen angesichts der zur Verfügung stehenden personellen und finanziellen Ressourcen ein hohes Ziel dar; die beschriebenen Kompetenzen entsprechen jedoch den Aufgaben von Ethikstrukturen. Auch wenn diese Ziele nicht sofort erfüllt werden können, sollen sie angestrebt werden.

10 Beispiele finden sich im Anhang zu den Empfehlungen «Ethische Unterstützung in der Medizin»; dieser ist auf der Website der SAMW zugänglich.

11 Slowther AM, Johnston C, Goodall J, Hope T. A practical guide for clinical ethics support. Oxford: The Ethox Centre. University of Oxford; 2004

12 Vgl. «Ausübung der ärztlichen Tätigkeit bei inhaftierten Personen». Medizin-ethische Richtlinien der SAMW.

\section{Literatur}

- Akademie für Ethik in der Medizin (AEM): Standards für Ethikberatung in Einrichtungen des Gesundheitswesens. Ethik Med. 22(2):149-153.

- American Society for Bioethics and Humanities (ASBH). Core Competencies for Health Care Ethics Consultation. Improving Competencies in Clinical Ethics Consultation: An Education Guide. 2009. (www.asbh. org/)

- Aulisio MP, Arnold RM, Youngner SJ. Health care ethics consultation: nature, goals, and competencies. A position paper from the Society for Health and Human Values-Society for Bioethics Consultation Task Force on Standards for Bioethics Consultation. Ann Intern Med. 2000;133(1):59-69.

- Baumann R, Arn C. Ethiktransfer in Organisationen: Basel: Schwabe AG; 2009.

- Bundesärztekammer, Zentrale Ethikkommission. Stellungnahme zur Ethikberatung in der klinischen Medizin vom 16. Juni 2006. (www.zentrale-ethikkommission.de/page. asp?his=0.1.18)

- Dörries A, Neitzke G, Simon A, Vollmann J (Hrsg.). Klinische Ethikberatung. Ein Praxisleitfaden für Krankenhäuser und Pflegeeinrichtungen. Stuttgart: Kohlhammer; 2008

- Gross D, May AT, Simon A (Hrsg.): Beiträge zur Klinischen Ethikberatung an Universitätskliniken. Berlin: LIT; 2008.

- Fournier V, Gaille-Nikodimov M. L'éthique clinique à l'Hôpital Cochin: une méthode à l'épreuve de l'expérience. Hôpital Cochin. 2007.

- Hurst SA, Reiter-Theil S, Baumann-Hölzle R, Foppa C, Malacrida R, Bosshard G, et al. The growth of clinical ethics in a multilingual country: challenges and opportunities. Bioethica Forum. 2008;1(1):15-24.

- Kettner M. Überlegungen zu einer integrierten Theorie von Ethik-Kommissionen und Ethik-Komitees. Honnefelder L, Streffer C (Hrsg.). Jahrbuch für Wissenschaft und Ethik C. 2002; (Vol 7):53-72. 
- Porz R, Rehmann-Sutter C, Scully JL, Zimmermann-Acklin M (Hrsg.).Gekauftes Gewissen? Zur Rolle der Bioethik in Institutionen. Mentis Paderborn. 2007.

- Salathé M, Amstad H, Jünger M, Leuthold M, Regamey C. Institutionalisierung der Ethikberatung an Akutspitälern, psychiatrischen Kliniken, Pflegeheimen und Einrichtungen der Rehabilitation der Schweiz: Zweite Umfrage der Schweizerischen Akademie der Medizinischen Wissenschaften. Bioethica Forum. 2008;1(1): 8-14.

- Slowther AM, Johnston C, Goodall J, Hope T. A practical guide for clinical ethics support. Oxford: The Ethox Centre, University of Oxford; 2004.

- Steinkamp N, Gordijn B. Ethical case deliberation on the ward. A comparison of four methods. Med Health Care Philos. 2003;6(3):235-46.

- Steinkamp N, Gordijn B. Ethik in Klinik und Pflegeeinrichtung. Ein Arbeitsbuch. 2. Aufl. Neuwied/Köln/München: Luchterhand; 2005.

- Vorstand der Akademie für Ethik in der Medizin e.V. Standards für Ethikberatung in Einrichtungen des Gesundheitswesens. Ethik Med. Vol 22.(2):149-53.(http://www. springerlink.com/content/ $9627261240864 \mathrm{vh} 8 /)$

\begin{tabular}{|c|c|}
\hline \multicolumn{2}{|c|}{ Hinweise zur Ausarbeitung dieser Empfehlungen } \\
\hline Auftrag & $\begin{array}{l}\text { Am 20. Juni } 2008 \text { hat die Zentrale Ethikkommission der SAMW eine Subkommis- } \\
\text { sion mit der Ausarbeitung von medizin-ethischen Empfehlungen betreffend } \\
\text { Strukturen zur Unterstützung der ethischen Reflexion beauftragt. }\end{array}$ \\
\hline $\begin{array}{l}\text { Verantwortliche } \\
\text { Subkommission }\end{array}$ & $\begin{array}{l}\text { Prof. Samia Hurst, Genf (Vorsitz) } \\
\text { Dr. Christof Arn, Scharans } \\
\text { Dr. Charles Chappuis, Spiegel } \\
\text { Dr. Carlo Foppa, Morges } \\
\text { Irma Graf, St. Gallen } \\
\text { Prof. Annemarie Kesselring, Bern } \\
\text { Prof. Christian Kind, St. Gallen, Präsident ZEK } \\
\text { Angelika Lehmann, BNS, MAS Ethik, Basel } \\
\text { Dr. Barbara Meyer-Zehnder, Basel } \\
\text { Dr. Rouven Porz, Bern } \\
\text { lic. iur. Michelle Salathé, MAE, Basel, stv. Generalsekretärin SAMW } \\
\text { Dr. Regula Schmitt, Köniz }\end{array}$ \\
\hline $\begin{array}{l}\text { Beigezogene } \\
\text { Experten }\end{array}$ & $\begin{array}{l}\text { PD Dr. Georg Bosshard, Winterthur } \\
\text { Jacques Butel, Genf } \\
\text { Dr. Marion Danis, Bethesda } \\
\text { Dr. Véronique Fournier, Paris } \\
\text { Dr. Tanja Krones, Zürich } \\
\text { Prof. Roberto Malacrida, Lugano } \\
\text { Dr. Gerald Neitzke, Hannover } \\
\text { Katrin Lanz, Spitex, Solothurn } \\
\text { Gabriela Sieger, Schweiz. Kinderspitex Verein, Horn }\end{array}$ \\
\hline Vernehmlassung & $\begin{array}{l}\text { Am 24. November } 2011 \text { hat der Senat der SAMW eine erste Fassung dieser } \\
\text { Richtlinien zur Vernehmlassung genehmigt. }\end{array}$ \\
\hline Genehmigung & $\begin{array}{l}\text { Die definitive Fassung dieser Richtlinien wurde am 29. Mai } 2012 \text { vom Senat der } \\
\text { SAMW genehmigt. }\end{array}$ \\
\hline
\end{tabular}

